\title{
ON THE $r$-TH HYPER-KLOOSTERMAN SUMS AND A PROBLEM OF D. H. LEHMER
}

\author{
Zhang Tianping And Xue Xifeng
}

\begin{abstract}
For any integer $k \geq 2$, let $P(c, k+1 ; q)$ be the number of all $k+1$-tuples with positive integer coordinates $\left(a_{1}, a_{2}, \ldots, a_{k+1}\right)$ such that $1 \leq a_{i} \leq q,\left(a_{i}, q\right)=1, a_{1} a_{2} \cdots a_{k+1} \equiv c(\bmod q)$ and $2 \nmid\left(a_{1}+a_{2}+\right.$ $\left.\cdots+a_{k+1}\right)$, and $E(c, k+1 ; q)=P(c, k+1 ; q)-\frac{\phi^{k}(q)}{2}$. The main purpose of this paper is using the properties of Gauss sums, primitive characters and the mean value theorems of Dirichlet $L$-functions to study the hybrid mean value of the $r$-th hyper-Kloosterman sums $K l(h, k+1, r ; q)$ and $E(c, k+1 ; q)$, and give an interesting mean value formula.
\end{abstract}

\section{Introduction}

Let $q \geq 3$ and $c$ be two integers with $(c, q)=1$. For each integer $a$ with $1 \leq a \leq q$ and $(a, q)=1$, we know that there exists one and only one $b$ with $1 \leq b \leq q$ such that $a b \equiv c(\bmod q)$. Let

$$
M(c, k ; q)=\sum_{\substack{a=1 \\ a b \equiv c(\bmod q)}}^{q} \sum_{b=1}^{q}(a-b)^{2 k},
$$

where $\sum_{a=1}^{\prime q}$ denotes the summation over all $a$ such that $(a, q)=1$. In [17], $\mathrm{W}$. Zhang used the estimates for Kloosterman sums and trigonometric sums to obtain a sharp asymptotic formula for $M(c, k ; q)$, and proved that for any positive integer $k$, we have the asymptotic formula

$$
M(c, k ; q)=\frac{1}{(2 k+1)(k+1)} \phi(q) q^{2 k}+O\left(4^{k} q^{\frac{4 k+1}{2}} d^{2}(q) \ln ^{2} q\right),
$$

where $\phi(q)$ is the Euler function, and $d(q)$ is the divisor function.

Received September 24, 2007; Revised March 18, 2008.

2000 Mathematics Subject Classification. 11L05.

Key words and phrases. $r$-th hyper-Kloosterman sums, hybrid mean value.

This work is supported by the NSF $(10671155,10871123)$ and PNSF (2007A06) of P. R. China.

(C)2009 The Korean Mathematical Society 
For any fixed positive integer $c$ with $(c, q)=1$, let

$$
N(c, 1 ; q)=M(c, 1 ; q)-\frac{1}{6} \phi(q) q^{2}-\frac{1}{3} q \prod_{p \mid q}(1-p),
$$

where $\prod_{p \mid q}$ denotes the product over all distinct prime divisors of $q$. W. Zhang [19] proved that

$$
\sum_{c=1}^{q} N^{2}(c, 1 ; q)=\frac{5}{36} q^{3} \phi^{3}(q) \prod_{p^{\alpha} \| q} \frac{\frac{(p+1)^{3}}{p\left(p^{2}+1\right)}-\frac{1}{p^{3 \alpha-1}}}{1+\frac{1}{p}+\frac{1}{p^{2}}}+O\left(q^{5} \exp \left(\frac{4 \ln q}{\ln \ln q}\right)\right),
$$

which shows that the error term in $(0.1)$ is the best possible.

Let $P(c ; q)$ be the number of cases in which $a$ and $\bar{a}$ are of opposite parity. For any odd prime $p$, D. H. Lehmer [3] asked us to find $P(1 ; p)$ or at least to say something nontrivial about it. In [16], W. Zhang proved that

$$
P(1 ; q)=\frac{1}{2} \phi(q)+O\left(q^{\frac{1}{2}} d^{2}(q) \ln ^{2} q\right) .
$$

Following the spirit of [19], [21] defined the error term

$$
E(c ; q)=M c ; q)-\frac{1}{2} \phi(q),
$$

and obtained that

$$
\sum_{c=1}^{p-1} E^{2}(c ; p)=\frac{3}{4} p^{2}+O\left(p \exp \left(\frac{3 \ln p}{\ln \ln p}\right)\right) .
$$

This proves the error terms in $(0.2)$ is also the best possible.

Moreover, W. Zhang [20] found that there exists some interesting connections between the error terms $E(c ; q)$ and the classical Kloosterman sums:

$$
K(m, n ; q)=\sum_{b=1}^{q} e\left(\frac{m b+n \bar{b}}{q}\right)
$$

where $e(y)=\mathrm{e}^{2 \pi i y}$, and obtained the hybrid mean value formula as

$$
\sum_{c=1}^{q} E(c ; q) K(\overline{4} c, 1 ; q)=\frac{4}{\pi^{2}} q \phi(q) \prod_{p \| q}\left(1-\frac{1}{p(p-1)}\right)+O\left(q^{\frac{3}{2}+\epsilon}\right),
$$

where $\epsilon$ is any fixed positive number. Note that here on the left hand side the term is of the size $O\left(q^{1+\epsilon}\right)$. The result in (0.3) shows that this term indeed has an average size of $O(q)$, and there is no significant cancellation occurred in this weighted sum. This is opposite to the standard belief that a sum of Kloosterman sums (without weighting by those error terms) of the type

$$
\sum_{q \leq x} \frac{K(m, n ; q)}{\sqrt{q}}
$$


should exhibit cancellation. Linnik [5] and Selberg [11] have conjectured that

$$
\sum_{q \leq x} \frac{K(m, n ; q)}{q}=O\left(x^{\epsilon}\right)
$$

for every $\epsilon>0$. This conjecture is still unproven, but Kuznetsov [4] has proved that it is true for $\epsilon>\frac{1}{6}$. For the details, see the excellent paper of Goldfeld and Sarnak [2], or Patterson [10] and references therein.

In reference [8], Mordell introduced the hyper-Kloosterman sums as following:

$$
K l(h, k+1 ; q)=\sum_{a_{1}=1}^{q} \sum_{a_{2}=1}^{q} \cdots \sum_{a_{k}=1}^{q} e\left(\frac{a_{1}+a_{2}+\cdots+a_{k}+h \cdot \bar{a}_{1} \bar{a}_{2} \cdots \bar{a}_{k}}{q}\right) .
$$

About the hyper-Kloosterman sums, many scholars had studied about it before. Applications of the hyper-Kloosterman sums were found in the estimation of Fourier coefficients of Maass forms [1] and the work on Selberg's eigenvalue conjecture [7]. On the other hand, Smith [12] had built some interesting connections between the hyper-Kloosterman sums and the Heibronn sums. And Ye [14] found certain identities between the hyper-Kloosterman sums and the twoterm exponential sums, which are in turn deduced from generalized DavenportHasse identities of Gauss sums.

Similarly, Zhang and the first author [15] defined the $r$-th hyper-Kloosterman sums as following:

$$
K l(h, k+1, r ; q)=\sum_{a_{1}=1}^{q} \sum_{a_{2}=1}^{q} \cdots \sum_{a_{k}=1}^{q} e\left(\frac{a_{1}^{r}+a_{2}^{r}+\cdots+a_{k}^{r}+h \cdot \bar{a}_{1}^{r} \bar{a}_{2}^{r} \cdots \bar{a}_{k}^{r}}{q}\right),
$$

and built some interesting connections between the $r$-th hyper-Kloosterman sums and the hyper Cochrane sums.

Let $P(c, k+1 ; q)$ be the number of all $(k+1)$-tuples with positive integer coordinates $\left(a_{1}, a_{2}, \ldots, a_{k+1}\right)$ such that $1 \leq a_{i} \leq q,\left(a_{i}, q\right)=1, a_{1} a_{2} \cdots a_{k+1} \equiv$ $c(\bmod q)$ and $2 \nmid\left(a_{1}+a_{2}+\cdots+a_{k+1}\right)$. That is,

$$
P(c, k+1 ; q)=\sum_{\substack{a_{1}=1 \\ a_{1} a_{2} \cdots a_{k+1} \equiv c(\bmod q) \\ 2 \nmid\left(a_{1}+a_{2}+\cdots+a_{k+1}\right)}}^{q} \sum_{\substack{a_{k+1}=1 \\ q}}^{\prime} 1 .
$$

In [6], Liu Huaning defined the error term as

$$
E(c, k+1 ; q)=P(c, k+1 ; q)-\frac{\phi^{k}(q)}{2},
$$

and proved that for any odd number $q \geq 3$ and integer $k \geq 2$, we have

$$
\sum_{c=1}^{q} E(c, k+1 ; q) K l\left(\overline{2}^{k} c, k+1 ; q\right)=\frac{(-4)^{k} q^{k} \phi(q)}{\pi^{k+1} i^{k+1}} \prod_{p \|_{q}}\left(1-\frac{p^{k}-1}{p^{k}(p-1)^{2}}\right)+O\left(q^{k+\epsilon}\right) \text {. }
$$


It is obvious that $K l(h, k+1, r ; q)=K l(h, k+1 ; q)$ if $d_{r} \equiv r \equiv 1(\bmod \phi(q))$, so we suppose that $d_{r}=r-\phi(q)\left[\frac{r}{\phi(q)}\right]>1$. In this paper, we shall use the properties of Gauss sums, primitive characters and the mean value theorems of Dirichlet $L$-functions to study the hybrid mean value of the $r$-th hyperKloosterman sums $K l(h, k+1, r ; q)$ and $E(c, k+1 ; q)$, and give an interesting mean value formula. That is, we shall prove the following:

Theorem. Let $q \geq 3$ be an odd number. Then for any positive integers $r, k$ with $d_{r}=r-\phi(q)\left[\frac{r}{\phi(q)}\right]>1$, we have the asymptotic formula

$\sum_{c=1}^{q} E(c, k+1 ; q) K l\left(\overline{2}^{k} c, k+1, r ; q\right)=\frac{(-4)^{k} q^{k} \phi(q)}{\pi^{k+1} i^{k+1}} \prod_{p \| q}\left(1-\frac{p^{k}-1}{p^{k}(p-1)^{2}}\right)+O\left(d_{r} q^{k+\frac{1}{2}+\epsilon}\right)$.

Remark. If we bound the left hand side by estimation of individual terms, we can use the bounds for the $r$-th hyper-Kloosterman sums $\left|K l\left(\overline{2}^{k} c, k+1, r ; q\right)\right|=$ $O\left((k+1) q^{\frac{k}{2}+\epsilon}\right)$ and the error term $|E(c, k+1, q)|=O\left(q^{\frac{k}{2}+\epsilon}\right)$. This will give us a bound for the left hand side $O\left(q^{k+1+\epsilon}\right)$, which is about the same as the bound in the theorem. This confirmed that the bound for the error term is best possible, and there is no major cancellation on the left side of (0.4).

Taking $q=p$ and $k=1$ in Theorem respectively, we may immediately deduce the following

Corollary 1. Let $p \geq 3$ be a prime. Then for any positive integers $r, k$ with $d_{r}=r-\phi(p)\left[\frac{r}{\phi(p)}\right]>1$, we have

$$
\sum_{c=1}^{p-1} E(c, k+1 ; p) K l\left(\overline{2}^{k} c, k+1, r ; p\right)=\frac{(-4)^{k} p^{k+1}}{\pi^{k+1} i^{k+1}}+O\left(d_{r} p^{k+\frac{1}{2}+\epsilon}\right) .
$$

Corollary 2. Let $q \geq 3$ be an odd number. Then for any positive integer $r$ with $d_{r}=r-\phi(q)\left[\frac{r}{\phi(q)}\right]>1$, we have

$$
\sum_{c=1}^{q} E(c, 2 ; q) K l(\overline{4} c, 2, r ; q)=\frac{4 q \phi(q)}{\pi^{2}} \prod_{p \|_{q}}\left(1-\frac{1}{p(p-1)}\right)+O\left(d_{r} q^{\frac{3}{2}+\epsilon}\right) .
$$

\section{Some lemmas}

To complete the proof of Theorem, we need the following several lemmas. Since the classical Gauss sums is defined as

$$
G(n, \chi ; q)=\sum_{b=1}^{q} \chi(b) e\left(\frac{n b}{q}\right)
$$

and $\tau(\chi)=G(1, \chi)$, we have the following: 
Lemma 1. For any positive integer $q$, let $\chi$ be a non-primitive character modulo $q$ and $\chi_{q} \Leftrightarrow \chi_{q^{*}}^{*}$. If $(n, q)>1$, we have

$$
\begin{aligned}
& G(n, \chi) \\
= & \left\{\begin{array}{cl}
\bar{\chi}^{*}\left(\frac{n}{(n, q)}\right) \chi^{*}\left(\frac{q}{q^{*}(n, q)}\right) \mu\left(\frac{q}{q^{*}(n, q)}\right) \phi(q) \phi^{-1}\left(\frac{q}{(n, q)}\right) \tau\left(\chi^{*}\right), & \text { if } q^{*}=\frac{q_{1}}{\left(n, q_{1}\right)} \\
0, & \text { if } q^{*} \neq \frac{q_{1}}{\left(n, q_{1}\right)},
\end{array}\right.
\end{aligned}
$$

where $\mu(n)$ is the Möbius function and $q_{1}$ is the greatest divisor of $q$ that has the same prime factors as $q^{*}$.

If $(n, q)=1$, then we have

$$
G(n, \chi)=\bar{\chi}^{*}(n) \chi^{*}\left(\frac{q}{q^{*}}\right) \mu\left(\frac{q}{q^{*}}\right) \tau\left(\chi^{*}\right) .
$$

Proof. See [9].

Lemma 2. Let $\chi$ be a character modulo $q$, generated by the primitive character $\chi_{m}$ modulo $m$. Then we have the identity

$$
\tau(\chi)=\chi_{m}\left(\frac{q}{m}\right) \mu\left(\frac{q}{m}\right) \tau\left(\chi_{m}\right) .
$$

Proof. See Lemma 1.3 of [9].

Lemma 3. Let $q \geq 3$ be an odd number and $k$ be a positive integer. Then for any positive integer $c$ with $(c, q)=1$, we have the identity

$$
E(c, k+1 ; q)=\frac{(-2)^{k}}{(\pi i)^{k+1} \phi(q)} \sum_{\substack{\chi \bmod q \\ \chi(-1)=-1}} \bar{\chi}(c)(1-2 \chi(2))^{k+1}\left(\sum_{n=1}^{\infty} \frac{G(n, \chi)}{n}\right)^{k+1}
$$

where $\chi$ denotes a Dirichlet character modulo $q$ with $\chi(-1)=-1$.

Proof. This is Lemma 1 of [6]. Since the paper is still in press, we state it out. From the orthogonality relation for character modulo $q$ we have

$$
\begin{aligned}
& P(c, k+1 ; q)=\frac{1}{2} \sum_{\substack{a_{1}=1 \\
a_{1} a_{2} \cdots a_{k+1} \equiv c(\bmod q)}}^{q} \sum_{a_{2}=1}^{q} \cdots \sum_{\substack{a_{k+1}=1 \\
(\bmod }}^{q}\left(1-(-1)^{a_{1}+a_{2}+\cdots+a_{k+1}}\right) \\
& =\frac{\phi^{k}(q)}{2}-\frac{1}{2} \sum_{\substack{a_{1}=1 \\
a_{1} a_{2} \cdots a_{k+1} \equiv c(\bmod q)}}^{q} \sum_{\substack{a_{2}=1 \\
a_{k+1}=1}}^{q}(-1)^{a_{1}+a_{2}+\cdots+a_{k+1}} \\
& =\frac{\phi^{k}(q)}{2}-\frac{1}{2 \phi(q)} \sum_{\chi \bmod } \bar{\chi}(c)\left[\sum_{a=1}^{q}(-1)^{a} \chi(a)\right]^{k+1} \text {. }
\end{aligned}
$$


Now if $\chi(-1)=1$, then

$$
\sum_{b=1}^{q}(-1)^{b} \chi(b)=0
$$

while if $\chi(-1)=-1$, then

$$
\sum_{b=1}^{q}(-1)^{b} \chi(b)=2 \chi(2) \sum_{b=1}^{(q-1) / 2} \chi(b) .
$$

From [13] we also know that for any odd character $\chi \bmod q$, we have

$$
(1-2 \chi(2)) \sum_{c=1}^{q} c \chi(c)=\chi(2) q \sum_{c=1}^{(q-1) / 2} \chi(c) .
$$

On the other hand, if $\chi(-1)=-1$, then

$$
\sum_{b=1}^{q} \chi(b)\left(\frac{b}{q}-\frac{1}{2}\right)=\frac{1}{q} \sum_{b=1}^{q} b \chi(b) .
$$

Thus we may immediately obtain

$$
\begin{aligned}
E(c, k+1 ; q) & =-\frac{1}{2 \phi(q)} \sum_{\substack{\chi \bmod q \\
\chi(-1)=-1}} \bar{\chi}(c)\left[\frac{2(1-2 \chi(2))}{q} \sum_{a=1}^{q} a \chi(a)\right]^{k+1} \\
& =-\frac{2^{k}}{\phi(q)} \sum_{\substack{\chi(-1)=-1 \\
\chi(\bmod q}} \bar{\chi}(c)(1-2 \chi(2))^{k+1}\left[\sum_{a=1}^{q} \chi(a)\left(\frac{a}{q}-\frac{1}{2}\right)\right]^{k} \\
& =-\frac{2^{k}}{\phi(q)} \sum_{\substack{\chi(-1)=-1 \\
\chi(-1)}} \bar{\chi}(c)(1-2 \chi(2))^{k+1}\left[\sum_{a=1}^{q} \chi(a)\left(\left(\frac{a}{q}\right)\right)\right]^{k+1} .
\end{aligned}
$$

Note that

$$
((x))=-\frac{1}{\pi} \sum_{n=1}^{\infty} \frac{\sin (2 \pi n x)}{n}, \quad \sin x=\frac{1}{2 i}\left(\mathrm{e}^{x i}-\mathrm{e}^{-x i}\right),
$$

therefore

$$
\begin{aligned}
\sum_{a=1}^{q} \chi(a)\left(\left(\frac{a}{q}\right)\right) & =\frac{-1}{\pi} \sum_{n=1}^{\infty} \frac{\sum_{a=1}^{q} \chi(a) \sin (2 \pi n a / q)}{n} \\
& =\frac{-1}{2 \pi i} \sum_{n=1}^{\infty} \frac{G(n, \chi)-G(-n, \chi)}{n}=\frac{-1}{\pi i} \sum_{n=1}^{\infty} \frac{G(n, \chi)}{n} .
\end{aligned}
$$


So from the above we have

$$
E(c, k+1 ; q)=\frac{(-2)^{k}}{(\pi i)^{k+1} \phi(q)} \sum_{\substack{\chi \bmod q \\ \chi(-1)=-1}} \bar{\chi}(c)(1-2 \chi(2))^{k+1}\left(\sum_{n=1}^{\infty} \frac{G(n, \chi)}{n}\right)^{k+1} .
$$

This proves Lemma 3 .

Lemma 4. Let $q$ and $r$ be integers with $q \geq 2$ and $(r, q)=1, \chi$ be a Dirichlet character modulo $q$. Then we have the identities

$$
\sum_{\bmod q}^{*} \chi(r)=\sum_{d \mid(q, r-1)} \mu\left(\frac{q}{d}\right) \phi(d)
$$

and

$$
J(q)=\sum_{d \mid q} \mu(d) \phi\left(\frac{q}{d}\right)
$$

where $\sum^{*} \chi \bmod q$ denotes the summation over all primitive characters modulo $q$ and $J(q)$ denotes the number of primitive characters modulo $q$.

Proof. This is Lemma 3 of [18].

Lemma 5. For any positive integers $q, r$, let $d_{r}=r-\phi(q)\left[\frac{r}{\phi(q)}\right]>1$ and $\chi_{1}$ be a $d_{r}$ th-order character modulo $q$. Then for any character $\chi$ modulo $q$, we have the identities

$$
\sum_{h=1}^{q} \bar{\chi}(h) K l(h, k+1,1 ; q)=\tau^{k+1}(\bar{\chi})
$$

and

$$
\sum_{h=1}^{q} \bar{\chi}(h) K l(h, k+1, r ; q)=\tau(\bar{\chi})\left(\tau(\bar{\chi})+\sum_{j=1}^{d_{r}-1} \tau\left(\bar{\chi} \chi_{1}^{j}\right)\right)^{k}
$$

Proof. This is Lemma 4 of [15].

Lemma 6. For any positive integers $r$ and $q \geq 3$ with $d_{r}=r-\phi(q)\left[\frac{r}{\phi(q)}\right]>1$, let $q=u v$, where $(u, v)=1$, $u$ be a square-full number or $u=1$, $v$ be a squarefree number, $\chi_{1}$ be a $d_{r}$ th-order character modulo $q$. Then we have

$$
\begin{aligned}
\Psi_{1}= & \sum_{d \mid v} \sum_{d_{1} \mid \frac{v}{d}} \cdots \sum_{d_{k+1} \mid \frac{v}{d}} \frac{(u d)^{k} \phi\left(d_{1}\right) \cdots \phi\left(d_{k+1}\right) \mu\left(d_{1}\right) \cdots \mu\left(d_{k+1}\right)}{d_{1} \cdots d_{k+1}} \sum_{\substack{\chi \bmod u d \\
\chi(-1)=-1}}^{*} \bar{\chi}\left(d_{1} \cdots d_{k+1}\right) \\
& \times L^{k+1}(1, \bar{\chi}) \sum_{a=1}^{u d} \chi(a) e\left(\frac{a}{u d}\right) \sum_{j=1}^{d_{r}-1} \sum_{l=0}^{q / u d-1} \sum_{m=1}^{u d} \chi_{1}^{j}(l u d+m) \bar{\chi}(m) e\left(\frac{l u d+m}{q}\right) \\
\ll & q^{k+\frac{3}{2}+\epsilon},
\end{aligned}
$$




$$
\begin{aligned}
\Psi_{2}= & \sum_{d \mid v} \sum_{d_{1} \mid \frac{v}{d}} \cdots \sum_{d_{k+1} \mid \frac{v}{d}} \frac{(u d)^{k} \phi\left(d_{1}\right) \cdots \phi\left(d_{k+1}\right) \mu\left(d_{1}\right) \cdots \mu\left(d_{k+1}\right)}{d_{1} \cdots d_{k+1}} \sum_{\substack{\chi \bmod u d \\
\chi(-1)=-1}}^{*} \bar{\chi}\left(d_{1} \cdots d_{k+1}\right) \\
& \times \bar{\chi}^{w}(2) L^{k+1}(1, \bar{\chi}) \sum_{a=1}^{u d} \chi(a) e\left(\frac{a}{u d}\right) \sum_{j=1}^{d_{r}-1} \sum_{l=0}^{q / u d-1} \sum_{m=1}^{u d} \chi_{1}^{j}(l u d+m) \bar{\chi}(m) e\left(\frac{l u d+m}{q}\right) \\
& \ll q^{k+\frac{3}{2}+\epsilon}, \quad w=1,2, \ldots, k+1 .
\end{aligned}
$$

Proof. Here we only prove the first estimate. Similarly, we can get another estimate.

Let $\tau_{k+1}(n)$ denote the $(k+1)$-th divisor function (i.e., the number of positive integer solutions of the equation $\left.n_{1} n_{2} \cdots n_{k+1}=n\right)$. Then for any parameter $N \geq u d$ and non-principal character $\chi$ modulo $u d$, applying Abel's identity we have

$$
L^{k+1}(1, \bar{\chi})=\sum_{n=1}^{\infty} \frac{\bar{\chi}(n) \tau_{k+1}(n)}{n}=\sum_{1 \leq n \leq N} \frac{\bar{\chi}(n) \tau_{k+1}(n)}{n}+\int_{N}^{\infty} \frac{A(y, \bar{\chi})}{y^{2}} \mathrm{~d} y,
$$

where $A(y, \bar{\chi})=\sum_{N<n \leq y} \bar{\chi}(n) \tau_{k+1}(n)$. Using Lemma 4 in [22] and Cauchy inequality, we have

$$
\sum_{\substack{\chi \bmod u d \\ \chi(-1)=-1}}|A(y, \bar{\chi})| \leq\left(\phi(u d) \sum_{\substack{\chi \bmod u d \\ \chi(-1)=-1}}|A(y, \bar{\chi})|^{2} \ll y^{1-\left(1 / 2^{k}\right)+\epsilon} \phi^{3 / 2}(u d) .\right.
$$

Hence we have

$$
\begin{aligned}
& \sum_{d \mid v} \sum_{d_{1} \mid \frac{v}{d}} \cdots \sum_{d_{k+1} \mid \frac{v}{d}} \frac{(u d)^{k} \phi\left(d_{1}\right) \cdots \phi\left(d_{k+1}\right) \mu\left(d_{1}\right) \cdots \mu\left(d_{k+1}\right)}{d_{1} \cdots d_{k+1}} \sum_{j=1}^{d_{r}-1} \sum_{l=0}^{q / u d-1} \sum_{m=1}^{u d} \chi_{1}^{j}(l u d+m) \\
& \times e\left(\frac{l u d+m}{q}\right) \sum_{a=1}^{u d} e\left(\frac{a m}{u d}\right) \sum_{\substack{\chi \bmod u d \\
\chi(-1)=-1}}^{*} \bar{\chi}\left(m d_{1} \cdots d_{k+1}\right) \chi(a m) \int_{N}^{\infty} \frac{A(y, \bar{\chi})}{y^{2}} d y \\
& \ll d_{r} q^{k+2+\epsilon} \int_{N}^{\infty} \frac{1}{y^{2}}\left(\sum_{\chi \bmod _{q}}|A(y, \bar{\chi})|\right) d y \ll d_{r} q^{k+2+\epsilon} \int_{N}^{\infty} \frac{y^{1-\frac{1}{2^{k}}+\epsilon} \phi^{3 / 2}(q)}{y^{2}} d y \\
& \ll \frac{d_{r} q^{k+\frac{7}{2}+\epsilon}}{N^{2-k}} .
\end{aligned}
$$

Combining the above we can get

$\Psi_{1}=\sum_{d \mid v} \sum_{d_{1} \mid \frac{v}{d}} \cdots \sum_{d_{k+1} \mid \frac{v}{d}} \frac{(u d)^{k} \phi\left(d_{1}\right) \cdots \phi\left(d_{k+1}\right) \mu\left(d_{1}\right) \cdots \mu\left(d_{k+1}\right)}{d_{1} \cdots d_{k+1}} \sum_{j=1}^{d_{r}-1} \sum_{l=0}^{q / u d-1} \sum_{m=1}^{u d} \chi_{1}^{j}(l u d+m)$ 
HYPER-KLOOSTERMAN SUMS AND A PROBLEM OF D. H. LEHMER

$$
\times e\left(\frac{l u d+m}{q}\right) \sum_{a=1}^{u d} e\left(\frac{a m}{u d}\right) \sum_{1 \leq n \leq N} \frac{\tau_{k+1}(n)}{n} \sum_{\substack{\chi \bmod u d \\ \chi(-1)=-1}}^{*} \bar{\chi}\left(n m d_{1} \cdots d_{k+1}\right) \chi(a m)+O\left(\frac{q^{k+\frac{7}{2}+\epsilon}}{N^{2-k}}\right) .
$$

Note that for any integer $a$ with $(a, q)=1$, from Lemma 4 we have

$$
\begin{aligned}
\sum_{\substack{\chi \bmod q \\
\chi(-1)=-1}}^{*} \chi(a) & =\frac{1}{2} \sum_{\chi}^{*}(1-\chi(-1)) \chi(a) \\
& =\frac{1}{2} \sum_{\chi}^{*} \chi(a)-\frac{1}{2} \sum_{\chi \bmod q}^{*} \chi(-a) \\
& =\frac{1}{2} \sum_{s \mid(q, a-1)} \mu\left(\frac{q}{s}\right) \phi(s)-\frac{1}{2} \sum_{s \mid(q, a+1)} \mu\left(\frac{q}{s}\right) \phi(s) .
\end{aligned}
$$

Therefore

$$
\begin{aligned}
& \Psi_{1}=\frac{1}{2} \sum_{d \mid v} \sum_{d_{1} \mid \frac{v}{d}} \cdots \sum_{d_{k+1} \mid \frac{v}{d}}(u d)^{k} \phi\left(d_{1}\right) \cdots \phi\left(d_{k+1}\right) \mu\left(d_{1}\right) \cdots \mu\left(d_{k+1}\right) \sum_{j=1}^{d_{r}-1} \sum_{l=0}^{q / u d-1} \sum_{m=1}^{u d} \chi_{1}^{j}(l u d+m) \\
& \times e\left(\frac{l u d+m}{q}\right) \sum_{a=1}^{u d} e\left(\frac{a m}{u d}\right) \sum_{n=1}^{N} \frac{\tau_{k+1}(n)}{n d_{1} \cdots d_{k+1}} \sum_{\substack{s \mid u d \\
n d_{1} \cdots d_{k+1} \equiv a(s)}} \mu\left(\frac{u d}{s}\right) \phi(s) \\
& -\frac{1}{2} \sum_{d \mid v} \sum_{d_{1} \mid \frac{v}{d}} \cdots \sum_{d_{k+1} \mid \frac{v}{d}}(u d)^{k} \phi\left(d_{1}\right) \cdots \phi\left(d_{k+1}\right) \mu\left(d_{1}\right) \cdots \mu\left(d_{k+1}\right) \sum_{j=1}^{d_{r}-1} \sum_{l=0}^{q / u d-1} \sum_{m=1}^{u d} \chi_{1}^{j}(l u d+m) \\
& \times e\left(\frac{l u d+m}{q}\right) \sum_{a=1}^{u d}{ }^{\prime} e\left(\frac{a m}{u d}\right) \sum_{n=1}^{N} \frac{\tau_{k+1}(n)}{n d_{1} \cdots d_{k+1}} \sum_{\substack{s \mid u d \\
n d_{1} \cdots d_{k+1} \equiv-a(s)}} \mu\left(\frac{u d}{s}\right) \phi(s) \\
& +O\left(\frac{q^{k+\frac{7}{2}+\epsilon}}{N^{2-k}}\right) \\
& =\frac{1}{2} \sum_{d \mid v} \sum_{d_{1} \mid \frac{v}{d}} \cdots \sum_{d_{k+1} \mid \frac{v}{d}}(u d)^{k} \phi\left(d_{1}\right) \cdots \phi\left(d_{k+1}\right) \mu\left(d_{1}\right) \cdots \mu\left(d_{k+1}\right) \sum_{j=1}^{d_{r}-1} \sum_{l=0}^{q / u d-1} \sum_{m=1}^{u d} \\
& \times \chi_{1}^{j}(l u d+m) e\left(\frac{l u d+m}{q}\right) \sum_{a=1}^{u d} e\left(\frac{\frac{q}{u d} \cdot a(l u d+m)}{q}\right) \sum_{n=1}^{N} \frac{\tau_{k+1}(n)}{a} \sum_{s \mid u d} \mu\left(\frac{u d}{s}\right) \phi \\
& +O\left(\sum_{d \mid v} \sum_{d_{1} \mid \frac{v}{d}} \cdots \sum_{d_{k+1} \mid \frac{v}{d}}(u d)^{k} \phi\left(d_{1}\right) \cdots \phi\left(d_{k+1}\right) \sum_{s \mid u d} \phi(s) \sum_{1 \leq l \leq \frac{N d_{1} \cdots d_{k+1}}{s}}(l s+a)^{\epsilon-1}\right) \\
& +O\left(\sum_{d \mid v} \sum_{d_{1} \mid \frac{v}{d}} \cdots \sum_{d_{k+1} \mid \frac{v}{d}}(u d)^{k} \phi\left(d_{1}\right) \cdots \phi\left(d_{k+1}\right) \sum_{s \mid u d} \phi(s) \sum_{1 \leq l \leq \frac{N d_{1} \cdots d_{k+1}}{s}}(l s-a)^{\epsilon-1}\right) \\
& +O\left(\frac{q^{k+\frac{7}{2}+\epsilon}}{N^{-k}}\right)
\end{aligned}
$$




$$
\begin{aligned}
= & \frac{1}{2} \sum_{d \mid v} \sum_{d_{1} \mid \frac{v}{d}} \cdots \sum_{d_{k+1} \mid \frac{v}{d}}(u d)^{k} \phi\left(d_{1}\right) \cdots \phi\left(d_{k+1}\right) \mu\left(d_{1}\right) \cdots \mu\left(d_{k+1}\right) \sum_{j=1}^{d_{r}-1} \sum_{b=1}^{q} \chi_{1}^{j}(b) e\left(\frac{b}{q}\right) \\
& \times \sum_{\substack{a=1 \\
d_{1} \cdots d_{k+1} \mid a}}^{u} \frac{\tau_{k+1}\left(a / d_{1} \cdots d_{k+1}\right)}{a} e\left(\frac{\frac{a v}{d} b}{q}\right) \sum_{s \mid u d} \mu\left(\frac{u d}{s}\right) \phi(s) \\
& +O\left(q^{k+1+\epsilon} N^{\epsilon_{1}}\right)+O\left(\frac{q^{k+\frac{7}{2}+\epsilon}}{N^{2-k}}\right)
\end{aligned}
$$

where we have used the estimate $\tau_{k+1}(n) \ll n^{\epsilon_{1}}$.

Now taking $N=q^{5 \cdot 2^{k-1}}$ on the above, and note that the identity $J(u)=$ $\phi^{2}(u) / u$ if $u$ is a square-full number, we can immediately obtain the following

$$
\begin{aligned}
\Psi_{1}= & \frac{1}{2} \sum_{d \mid v} \sum_{d_{1} \mid \frac{v}{d}} \cdots \sum_{d_{k+1} \mid \frac{v}{d}}(u d)^{k} J(u d) \phi\left(d_{1}\right) \cdots \phi\left(d_{k+1}\right) \mu\left(d_{1}\right) \cdots \mu\left(d_{k+1}\right) \\
& \times \sum_{\substack{a=1 \\
d_{1} \cdots d_{k+1} \mid a}}^{\prime \prime} \frac{\tau_{k+1}\left(a / d_{1} \cdots d_{k+1}\right)}{a} \sum_{j=1}^{d_{r}-1} G\left(1+a v / d, \chi_{1}^{j}\right)+O\left(q^{k+1+\epsilon}\right) \\
\ll & \sum_{d \mid v} \sum_{d_{1} \mid \frac{v}{d}} \cdots \sum_{d_{k+1} \mid \frac{v}{d}}(u d)^{k} J(u d) \phi\left(d_{1}\right) \cdots \phi\left(d_{k+1}\right) \\
& \times \sum_{a=1}^{u d} \frac{\tau_{k+1}\left(a / d_{1} \cdots d_{k+1}\right)}{a} \sum_{j=1}^{d_{r}-1}\left|G\left(1+a v / d, \chi_{1}^{j}\right)\right|+O\left(q^{k+1+\epsilon}\right) \\
\ll & \sum_{d \mid v} \sum_{d_{1} \mid \frac{v}{d}} \cdots \sum_{d_{k+1} \mid \frac{v}{d}}(u d)^{k} J(u d) \phi\left(d_{1}\right) \cdots \phi\left(d_{k+1}\right) d_{r} q^{\frac{1}{2}+\epsilon} \sum_{a=1}^{u d} \frac{(1+a v / d, q)}{a}+O\left(q^{k+1+\epsilon}\right) \\
\ll & d_{r} q^{\frac{1}{2}+\epsilon} \sum_{d \mid v} \sum_{d_{1} \mid \frac{v}{d}} \cdots \sum_{d_{k+1} \mid \frac{v}{d}}(u d)^{k+1} \phi\left(d_{1}\right) \cdots \phi\left(d_{k+1}\right) \sum_{s \mid q} \sum_{a=1}^{u d} \frac{s}{a}+O\left(q^{k+1+\epsilon}\right) \\
\ll & d_{r} q^{k+\frac{3}{2}+\epsilon},
\end{aligned}
$$

where we have used the properties of Gauss sums that

$$
\left|G\left(1+a v / d, \chi_{1}^{j}\right)\right| \ll(1+a v / d, q) q^{\frac{1}{2}+\epsilon}
$$

in the case that $\chi_{1}^{i}$ is not principal character modulo $q$. This completes the proof of Lemma 6 .

\section{Proof of Theorem}

In this section, we shall complete the proof of Theorem. Let $q \geq 3$ be an odd number. Then for any positive integers $r, k$ with $d_{r}=r-\phi(q)\left[\frac{r}{\phi(q)}\right]>1$ 
and any character $\chi \bmod q$, we have

$$
\begin{aligned}
& \sum_{c=1}^{q} \bar{\chi}(c) K l\left(\overline{2}^{k} c, k+1, r ; q\right) \\
= & \sum_{a_{1}=1}^{q} \sum_{a_{2}=1}^{q} \cdots \sum_{a_{k+1}=1}^{\prime} \sum_{c=1}^{q} \bar{\chi}(c) \mathrm{e}\left(\frac{a_{1}^{r}+a_{2}^{r}+\cdots+a_{k}^{r}+\overline{2}^{k} c \cdot \bar{a}_{1}^{r} \bar{a}_{2}^{r} \cdots \bar{a}_{k}^{r}}{q}\right) \\
= & \bar{\chi}^{k+1}(2) \tau(\bar{\chi})\left(\tau(\bar{\chi})+\sum_{j=1}^{d_{r}-1} \tau\left(\bar{\chi} \chi_{1}^{j}\right)\right)^{k} .
\end{aligned}
$$

Hence from Lemma 3 we may have

$$
\begin{aligned}
& \sum_{c=1}^{q} E(c, k+1 ; q) K l\left(\overline{2}^{k} c, k+1, r ; q\right) \\
& =\frac{(-2)^{k}}{(\pi i)^{k+1} \phi(q)} \sum_{\substack{\chi \bmod q \\
\chi(-1)=-1}}\left[\sum_{c=1}^{q} \bar{\chi}(c) K l\left(\overline{2}^{k} c, k+1, r ; q\right)\right](1-2 \chi(2))^{k+1}\left(\sum_{n=1}^{\infty} \frac{G(n, \chi)}{n}\right)^{k+1} \\
& =\frac{(-2)^{k}}{(\pi i)^{k+1} \phi(q)} \sum_{\substack{\chi \bmod q \\
\chi(-1)=-1}}(\bar{\chi}(2)-2)^{k+1} \tau(\bar{\chi})\left(\tau(\bar{\chi})+\sum_{j=1}^{d_{r}-1} \tau\left(\bar{\chi} \chi_{1}^{j}\right)\right)^{k}\left(\sum_{n=1}^{\infty} \frac{G(n, \chi)}{n}\right)^{k+1} \\
& =\sum_{c=1}^{q} E(c, k+1 ; q) K l\left(\overline{2}^{k} c, k+1 ; q\right) \\
& +\frac{k(-2)^{k}}{(\pi i)^{k+1} \phi(q)} \sum_{\substack{\chi \bmod q \\
\chi(-1)=-1}}(\bar{\chi}(2)-2)^{k+1} \tau^{k}(\bar{\chi})\left(\sum_{j=1}^{d_{r}-1} \tau\left(\bar{\chi} \chi_{1}^{i}\right)\right)\left(\sum_{n=1}^{\infty} \frac{G(n, \chi)}{n}\right)^{k+1} \\
& +\frac{(-2)^{k}}{(\pi i)^{k+1} \phi(q)} \sum_{t=2}^{k} C_{k}^{t} \sum_{\substack{\chi \bmod _{q} \\
\chi(-1)=-1}}(\bar{\chi}(2)-2)^{k+1} \tau^{k-t+1}(\bar{\chi})\left(\sum_{j=1}^{d_{r}-1} \tau\left(\bar{\chi} \chi_{1}^{j}\right)\right)^{t}\left(\sum_{n=1}^{\infty} \frac{G(n, \chi)}{n}\right)^{k+1} \\
& \equiv \sum_{c=1}^{q} E(c, k+1 ; q) K l\left(\overline{2}^{k} c, k+1 ; q\right)+E_{1}+E_{2},
\end{aligned}
$$

where $C_{k}^{t}=\frac{k !}{(k-t) ! t !}$.

We now let $q=u v$, where $(u, v)=1, u$ be a square-full number or $u=1, v$ be a square-free number. Note that $\chi^{*}\left(\frac{q}{m}\right) \mu\left(\frac{q}{m}\right) \neq 0$ if and only if $m=u d$, 
where $d \mid v$. So from Lemma 1, Lemma 2 and Lemma 3 we have

$$
\begin{aligned}
E_{1}= & \frac{k(-2)^{k}}{(\pi i)^{k+1} \phi(q)} \sum_{\substack{\chi \bmod _{q} \\
\chi(-1)=-1}}(\bar{\chi}(2)-2)^{k+1} \tau^{k}(\bar{\chi})\left(\sum_{j=1}^{d_{r}-1} \tau\left(\bar{\chi} \chi_{1}^{i}\right)\right)\left(\sum_{n=1}^{\infty} \frac{G(n, \chi)}{n}\right)^{k+1} \\
= & \frac{k(-2)^{k}}{(\pi i)^{k+1} \phi(q)} \sum_{d \mid v} \sum_{\substack{\chi(-1)=-1 \\
\text { mod }}}^{*}(\bar{\chi}(2)-2)^{k+1} \bar{\chi}^{k}\left(\frac{v}{d}\right) \mu^{k}\left(\frac{v}{d}\right) \tau^{k}(\bar{\chi}) \\
& \times\left(\sum_{d_{1} \mid \frac{v}{d}} \frac{\phi(q) \chi\left(\frac{v}{d d_{1}}\right) \mu\left(\frac{v}{d d_{1}}\right) \tau(\chi) L(1, \bar{\chi})}{d_{1} \phi\left(\frac{q}{d_{1}}\right)}\right)^{k+1}\left(\sum_{j=1}^{d_{r}-1} \tau\left(\bar{\chi} \chi_{1}^{i}\right)\right) \\
= & \frac{k \cdot 2^{k}}{(\pi i)^{k+1} \phi(q)} \sum_{d \mid v} \sum_{d_{1} \mid \frac{v}{d}} \cdots \sum_{d_{k+1} \mid \frac{v}{d} \chi} \sum_{\bmod u d}^{*} \frac{u^{k} d^{k} \phi\left(d_{1}\right) \cdots \phi\left(d_{k+1}\right) \mu\left(d_{1}\right) \cdots \mu\left(d_{k+1}\right)}{d_{1} \cdots d_{k+1}} \\
& \left((-2)^{k+1}+\sum_{w=1}^{k+1} \bar{\chi}^{w}(2)(-2)^{k-w+1}\right) \times \bar{\chi}\left(d_{1} \cdots d_{k+1}\right) L^{k+1}(1, \bar{\chi}) \sum_{a=1}^{u d} \chi(a) e\left(\frac{a}{u d}\right) \sum_{j=1}^{d_{r}-1} \sum_{l=0}^{q / u d-1} \\
& \times \sum_{m=1}^{u d} \chi_{1}^{j}(l u d+m) \bar{\chi}(m) e\left(\frac{l u d+m}{q}\right),
\end{aligned}
$$

where we have used the identities that

$\bar{\chi}\left(\frac{v}{d}\right)=\bar{\chi}\left(\frac{v}{d d_{1}}\right) \bar{\chi}\left(d_{1}\right), \quad \mu\left(\frac{v}{d}\right)=\mu\left(\frac{v}{d d_{1}}\right) \mu\left(d_{1}\right), \quad \phi(q)=\phi\left(\frac{q}{d_{1}}\right) \phi\left(d_{1}\right)$,

and

$$
\tau\left(\chi^{*}\right) \tau\left(\bar{\chi}^{*}\right)=-m
$$

where $\chi^{*}$ is a primitive character modulo $m$ and $\chi(-1)=-1$. So from Lemma 6 we have the estimate

$$
E_{1} \ll d_{r} q^{k+\frac{1}{2}+\epsilon} .
$$

Using the similar method of proving Lemma 6 , we may obtain the following estimate

$$
E_{2} \ll d_{r} q^{k+\frac{1}{2}+\epsilon} .
$$

Therefore from (0.3), (0.4) and (0.5), we may immediately obtain

$$
\begin{aligned}
& \sum_{c=1}^{q} E(c, k+1 ; q) K l\left(\overline{2}^{k} c, k+1, r ; q\right) \\
= & \sum_{c=1}^{q} E(c, k+1 ; q) K l\left(\overline{2}^{k} c, k+1 ; q\right)+O\left(d_{r} q^{k+\frac{1}{2}+\epsilon}\right) \\
= & \frac{(-4)^{k} q^{k} \phi(q)}{\pi^{k+1} i^{k+1}} \prod_{p \| q}\left(1-\frac{p^{k}-1}{p^{k}(p-1)^{2}}\right)+O\left(d_{r} q^{k+\frac{1}{2}+\epsilon}\right) .
\end{aligned}
$$


Acknowledgments. The authors express their gratitude to the referee for careful reading of the manuscript and many valuable suggestions.

\section{References}

[1] D. Bump, W. Duke, J. Hoffstein, and H. Iwaniec, An estimate for the Hecke eigenvalues of Maass forms, Internat. Math. Res. Notices (1992), no. 4, 75-81.

[2] D. Goldfeld and P. Sarnak, Sums of Kloosterman sums, Invent. Math. 71 (1983), no. 2, $243-250$.

[3] R. K. Guy, Unsolved Problems in Number Theory, Second edition. Problem Books in Mathematics. Unsolved Problems in Intuitive Mathematics, I. Springer-Verlag, New York, 1994.

[4] N. V. Kuznetsov, The Petersson conjecture for cusp forms of weight zero and the Linnik conjecture. Sums of Kloosterman sums, Mat. Sb. (N.S.) 111(153) (1980), no. 3, 334$383,479$.

[5] J. V. Linnik, Additive problems and eigenvalues of the modular operators, Proc. Internat. Congr. Mathematicians (Stockholm, 1962) pp. 270-284 Inst. Mittag-Leffler, Djursholm, 1963.

[6] H. Liu, On a problem of D. H. Lehmer and hyper-Kloosterman sums, Adv. Math. (China) 36 (2007), no. 2, 245-252.

[7] W. Luo, Z. Rudnick, and P. Sarnak, On Selberg's eigenvalue conjecture, Geom. Funct. Anal. 5 (1995), no. 2, 387-401.

[8] L. J. Mordell, On a special polynomial congruence and exponential sum, Calcutta Math. Soc. Golden Jubilee Commemoration Vol. pp. 29-32 Calcutta Math. Soc., Calcutta, 1963.

[9] C. Pan and C. Pan, Goldbach Conjecture, Science Press, Beijing, 1992.

[10] S. J. Patterson, The asymptotic distribution of exponential sums. I, Experiment. Math. 12 (2003), no. 2, 135-153.

[11] A. Selberg, On the estimation of Fourier coefficients of modular forms, Proc. Sympos. Pure Math., Vol. VIII pp. 1-15 Amer. Math. Soc., Providence, R.I., 1965.

[12] R. A. Smith, On n-dimensional Kloosterman sums, J. Number Theory 11 (1979), no. 3 S. Chowla Anniversary Issue, 324-343.

[13] F. Takeo, On Kronecker's limit formula for Dirichlet series with periodic coefficients, Acta Arith. 55 (1990), no. 1, 59-73.

[14] Y. Ye, Estimation of exponential sums of polynomials of higher degrees. II, Acta Arith. 93 (2000), no. 3, 221-235.

[15] T. Zhang and W. Zhang, On the $r$-th hyper-Kloosterman sums and its hybrid mean value, J. Korean Math. Soc. 43 (2006), no. 6, 1199-1217.

[16] W. Zhang, A problem of D. H. Lehmer and its generalization. II, Compositio Math. 91 (1994), no. 1, 47-56.

[17] _ On the difference between an integer and its inverse modulo $n$, J. Number Theory 52 (1995), no. 1, 1-6.

[18] _ On a Cochrane sum and its hybrid mean value formula, J. Math. Anal. Appl. 267 (2002), no. 1, 89-96.

[19] _ On the difference between an integer and its inverse modulo $n$. II, Sci. China Ser. A 46 (2003), no. 2, 229-238.

[20] - On a problem of D. H. Lehmer and Kloosterman sums, Monatsh. Math. 139 (2003), no. 3, 247-257.

[21] A problem of D. H. Lehmer and its mean square value formula, Japan. J. Math. (N.S.) 29 (2003), no. 1, 109-116

[22] W. Zhang, Y. Yi, and X. He, On the $2 k$-th power mean of Dirichlet L-functions with the weight of general Kloosterman sums, J. Number Theory 84 (2000), no. 2, 199-213. 
Zhang TianPing

College of Mathematics and Information Science

ShannXi Normal University

Xi'An, Shathxi, P. R. China

AND

Department of Mathematics

Northwest University

Xi'an, ShaAnxi, P. R. China

E-mail address: tpzhang@snnu.edu.cn

Xue Xifeng

Department of Mathematics

Northwest University

Xi'An, ShaAnxi, P. R. China

E-mail address: x_f_xue@126.com 